\title{
Mechanisms of androgen receptor repression in prostate cancer
}

Powell, S. M., Whitaker, H. C., Brooke, G. N., Gamble, S. C., Chotai, D., Dart, D. A., Belandia, B. and Bevan, C. L.

Department of Oncology, Imperial College London, Hammersmith Hospital, London W12 0NN.

${ }^{1}$ University of Cambridge, ${ }^{2}$ Instituto de Investigaciones Biomédicas.

Key words: androgen receptor, prostate cancer, antiandrogens, corepressors 


\begin{abstract}
Antiandrogens used in prostate cancer therapy inhibit androgen receptor activity via largely unknown mechanisms. Although initially successful in most cases, they eventually fail and the disease progresses. We need to elucidate how antiandrogens work to understand why they fail, and prolong their effects or design further therapies.

Using a cellular model we found different antiandrogens have diverse effects on subcellular localization of androgen receptor, revealing that they work via different mechanisms and suggesting that an informed sequential treatment regime may benefit patients. In the presence of the antiandrogens bicalutamide and hydroxyflutamide a significant proportion of the androgen receptor is translocated to the nucleus but remains inactive. Receptor inhibition under these conditions is likely to involve recruitment of corepressor proteins, which interact with antagonist-occupied receptor but inhibit receptor-dependent transcription. Which corepressors are required in vivo for androgen receptor repression by antiandrogens is not clear, but one candidate is the Notch effector Hey1. This inhibits ligand-dependent activity of the androgen receptor but not other steroid receptors. Further, it is excluded from the nucleus in most human prostate cancers, suggesting that abnormal subcellular distribution of corepressors may contribute to the aberrant hormonal responses observed in prostate cancer. A decrease in corepressors is one possible explanation for the development of antiandrogen-resistant prostate cancer, and this suggests that it may not occur at the gross level of protein expression.
\end{abstract}




\section{Background}

The androgen signalling pathway is key in prostate cancer progression and therapy and hence repressing it is the major aim of therapy. Prostate tumours, like the prostate itself, are dependent on circulating androgens for growth hence the gold standard for their treatment is orchidectomy or chemical castration, which ablate testicular androgens. However, the adrenal secretes weak androgens that can be converted to testosterone and subsequently the potent dihydrotestosterone, resulting in relatively high intracellular levels of the latter in the prostate (around 60\% non-castrate levels) [1]. Antiandrogens are thus often used to oppose the actions of residual androgens. This treatment is effective in around $80 \%$ of cases, but the majority of patients relapse after $1-2$ years. This advanced, hormone-refractory stage of the disease is aggressive and metastatic and currently there are no effective therapies for it. The mechanisms of progression to hormone-resistant disease are not fully understood, although many hypotheses have been put forward most of which involve alterations in the AR signalling pathway [2].

The androgen receptor (AR) is a ligand-activated transcription factor and a member of the nuclear receptor superfamily [3]. In the absence of ligand it is cytoplasmic; existing in a complex containing heatshock proteins, which are believed to hold the AR in a ligand binding-competent, inactive conformation. On ligand binding these proteins were thought to dissociate, although recent evidence indicates that even nuclear steroid receptors are associated with heat shock proteins [4]. The receptor dimerises and translocates into the nucleus where it bind to response elements (AREs) in the promoters of target genes and alters the rate of transcription from these promoters. Increasing transcription requires the 
recruitment of accessory proteins called coactivators while inhibiting transcription may require the recruitment of corepressor proteins under some circumstances [5].

\section{How do antiandrogens inhibit androgen receptor activity?}

Antiandrogens, which may be steroidal in structure (e.g. cyproterone acetate) or nonsteroidal (e.g. hydroxyflutamide, bicalutamide), bind to the ligand-binding domain of the AR but inhibit AR activity. The mechanism(s) by which they do this are not well understood. Since they are a mainstay of prostate cancer therapy it is imperative that every effort is made to understand how they work and hence why they fail, in a bid to delay or reverse the failure of such therapies. Figure 1 shows the various steps in the AR activation pathway at which antiandrogens could exert their effects. Competition with ligand for binding (i) undoubtedly contributes but cannot entirely account for antiandrogen action since the relative binding affinities of the AR for antiandrogens (compared to that for androgens) are very low. Inhibition of translocation into the nucleus or increased nuclear export, resulting in cytoplasmic accumulation of the receptor (ii), has been shown for the antioestrogen faslodex, used in breast cancer therapy [6]. However it is known that certain antiandrogens do promote nuclear accumulation of the AR. Recently inhibition of DNA-binding (iii) by bicalutamide and hydroxyflutamide was reported [7]. More downstream events such as the recruitment of cofactor proteins are also likely to be affected (iv). For instance, Masiello et al showed by chromatin immunoprecipitation that AR bound to bicalutamide can bind to DNA but instead of recruiting coactivator proteins, the corepressor protein NCoR was found at the ARE [8]. 
We undertook a cell-based study to compare the three most commonly used antiandrogens and determine their effects on AR localisation using in situ cell fractionation followed by immunoblotting [9]. The cells used were the PC3 cell line, which is negative for AR expression, stably transfected with AR expression plasmid, resulting in AR expression levels comparable to those seen in the LNCaP line, which has endogenous AR. This model was preferred to LNCaP cells since the AR in LNCaP cells has an amino acid substitution that allows activation of the receptor by antiandrogens. The results are summarised in Table 1. Treatment with cyproterone acetate resulted in AR accumulation in the cytoplasm. Hydroxyflutamide and bicalutamide both promoted nuclear entry but surprisingly we saw the AR largely in the subnuclear fraction associated with the nuclear matrix, rather than in the nucleoplasm as was the case in the presence of androgen. It is possible that AR in the nuclear matrix fraction is less likely to bind to AREs, thus the antiandrogens are effectively reducing DNA-binding. Alternatively, it may be that the AR is in this fraction s a result of binding to proteins that differ to those it binds in the presence of ligand, as suggested by the chromatin IP experiments of Masiello et al [8]. The isolation and identification of the AR-associated proteins in the different cellular fractions after treatment with ligand and antiandrogens will help to elucidate how these antiandrogens are exerting their effects.

\section{Are corepressors the answer?}

Corepressors were originally identified as proteins that are recruited to DNA-bound nuclear receptors such as thyroid hormone receptor in the absence of ligand and inhibit basal transcription from the target promoters [5]. Steroid receptors generally do not bind 
DNA in the absence of ligand, but evidence is increasing that they may recruit inhibitor corepressors in the presence of antagonist. For instance, direct interaction has been shown between the progesterone receptor and the corepressor $\mathrm{NCoR}$ in the presence of the antiprogestin RU486 [10]; and while a direct antiandrogen-dependent interaction between the AR and NCoR has not been demonstrated they do coexist on an ARE in the presence of bicalutamide [8] and interact in the presence of RU486 and DHT [11]. Thus it seems likely that nuclear AR-associated proteins in the presence of antiandrogens will include known and/or novel corepressors. It is not yet clear which corepressors are important in vivo for AR regulation in the prostate. No naturally occurring antiandrogens have yet been identified, and conceptually it seems unlikely that corepressor proteins exist that will only interact with the AR in the presence of a pharmaceutical antiandrogen. Hence it is unsurprising that, of the AR corepressors identified so far, most of them seem to also inhibit ligand-induced AR activity. This may be a mechanism to switch off or attenuate activated receptor signalling, allowing the cell to respond to changes in hormone levels. Further, androgens affect many target tissues, including bone, hair follicles and neurones as well sex organs. Different cells exposed to similar levels of hormone may be required to respond differently, activating different subsets of genes, and corepressor proteins could be involved in such "fine-tuning" of the hormonal response. The mechanisms by which corepressors reduce AR activity vary and those identified to date include recruitment of histone deacetylase activity, and functional or physical competition with coactivators. The corepressor Heyl, which is an effector of the Notch signalling pathway, appears to use both of these and has an HDAC-dependent and an HDAC-independent inhibitory effect on AR activity [12]. 


\section{Why do antiandrogens fail?}

Resistance to antiandrogen therapy is the major clinical problem in prostate cancer. Understanding and overcoming the progression of tumours to this stage will require the elucidation of how antiandrogens work. A popular hypothesis is that a somatic increase in coactivator proteins, or decrease in corepressors, may drive higher AR activity and hence tumour growth even at low, castrate levels of androgens. If corepressors are indeed required for antiandrogens to inhibit AR activity, a reduction in corepressor levels could also drive antiandrogen resistance. However, as yet there is little convincing evidence for demonstrable alterations in the levels of cofactors in prostate cancer. Several expression studies have shown small changes supportive of the idea, but others show no significant differences in benign versus malignant tissue [13-15]. It may be that the relevant cofactors are not being examined, and may not even yet be identified. It may be that differences are not evident at the RNA level, and will only be evident at the protein level. To go further, any changes may not be at the gross level of protein expression, but rather at the coding and/or functional level. In support of this, we found that levels of the AR corepressor Heyl were not noticably altered in prostate cancer versus benign prostate tissue, using immunohistochemistry. However, while immunohistochemistry is not a reliably quantitative technique, it allows visualisation of the protein localisation within the cell, and we saw that the distribution of Heyl was altered in the cancer samples [12]. Whereas in normal and benign tissue Heyl showed mainly nuclear localisation, in 8/10 prostate cancer samples it was exclusively cytoplasmic (Figure 2). This nuclear exclusion would effectively reduce functional corepressor levels, since the nucleus is where 
corepressors act. Thus while cytoplasmic sequestration of the AR itself is one mechanism of action of antiandrogens, cytoplasmic sequestration of corepressors is a possible mechanism of escape from antiandrogen therapy.

\section{Clinical implications}

At present, when a prostate cancer patient relapses on antiandrogen therapy treatment options are limited. Withdrawal of the antiandrogen, seemingly paradoxically, may result in a short improvement in symptoms, possibly because of an adaptive mutation in the AR causing its activation by the antiandrogen [2]. However, tumour growth will soon recur and often only palliative chemotherapy can be offered. It is not common practise to switch to treatment with a second antiandrogen after one has failed. We found that the mechanisms of action, and perhaps the cofactor proteins involved, are different for different antiandrogens. Such studies could be used as the basis for a rational sequence of antiandrogens in treatment thus prolonging the relapse-free period and life expectancy. For instance, failure of flutamide therapy may not preclude a response to cyproterone acetate. This has already been established in breast cancer, where treatment of tamoxifen-resistant patients with the second antioestrogen faslodex leads to clinical benefit in around $40 \%$ of cases [16].

Many studies are examining the levels of coactivators and corepressors in prostate cancer tissue, but convincing changes are few and far between. However, alterations occurring at the functional level may be visible only if studies are carried out at the cellular level. Our small -scale study revealed that Hey1 is excluded from the nucleus in prostate. If our further studies indicate that this is functionally significant in prostate 
cancer, future therapies could be based on reversing such aberrant localisation with a view to re-establishing sensitivity to antiandrogens.

1. Labrie, F. (1991) Endocrinol Metab Clin North Am 20, 845-72.

2. Feldman, B.J. and D. Feldman (2001) Nat Rev Cancer 1, 34-45.

3. Mangelsdorf, D.J., et al. (1995) Cell 83, 835-839.

4. Knoblauch, R. and M.J. Garabedian (1999) Mol Cell Biol 19, 3748-59.

5. McKenna, N.J., R.B. Lanz, and B.W. O'Malley (1999) Endocrine Rev 20, 321344.

6. Dauvois, S., R. White, and M.G. Parker (1993) J Cell Sci 106, 1377-88.

7. Farla, P., et al. (2005) J Cell Sci 118, 4187-98.

8. Masiello, D., et al. (2002) J Biol Chem 277, 26321-6.

9. Whitaker, H.C., et al. (2004) Clin Cancer Res 10, 7392-401.

10. Jackson, T.A., et al. (1997) Mol. Endo. 11, 693-705.

11. Hodgson, M.C., et al. (2005) J Biol Chem 280, 6511-9.

12. Belandia, B., et al. (2005) Mol Cell Biol 25, 1425-36.

13. Fujimoto, N., et al. (2001) Urology 58, 289-94.

14. Linja, M.J., et al. (2004) Clin Cancer Res 10, 1032-40.

15. Culig, Z., et al. (2004) J Steroid Biochem Mol Biol 92, 265-71.

16. Morris, C. and A. Wakeling (2002) Endocr Relat Cancer 9, 267-76. 
Table 1. Androgen receptor targeting by androgen and antiandrogens

\begin{tabular}{|c|c|c|c|c|}
\hline \multicolumn{5}{|c|}{ Treatment } \\
\hline $\begin{array}{c}\text { Vehicle } \\
\text { (Ethanol) }\end{array}$ & $\begin{array}{c}\text { Androgen } \\
\text { (Mibolerone) }\end{array}$ & Hydroxyflutamide & Bicalutamide & $\begin{array}{c}\text { Cyproterone } \\
\text { acetate }\end{array}$ \\
\hline $\mathrm{C}>>\mathrm{N}$ & $\mathrm{N}>>\mathrm{C}$ & $\mathrm{NM}>\mathrm{C}>>\mathrm{N}$ & $\mathrm{NM}>\mathrm{C}>>\mathrm{N}$ & $\mathrm{CM}=\mathrm{C}>>\mathrm{N}$ \\
\hline
\end{tabular}

$\mathrm{C}=$ cytoplasmic $; \mathrm{N}=$ nucleoplasm, $\mathrm{NM}=$ nuclear matrix $; \mathrm{CM}=$ cytoplasmic membraneassociated 


\section{Figure legends}

Figure 1. The androgen receptor activation pathway, showing steps at which

antiandrogens might act. $\mathrm{AR}=$ androgen receptor, $\mathrm{ARE}=$ androgen response element, HSP $=$ heatshock protein

Figure 2. Nuclear exclusion of Hey1 in human prostate cancer cells. Sections of formalinfixed human prostate tissue from patients with benign prostatic hyperplasia or malignant adenocarcinoma as indicated were stained with anti-Hey1 antibody Brown color reflects positive staining for Hey1, negative nuclei show blue color. 


\section{Figure 1}

androgen

antiandrogen

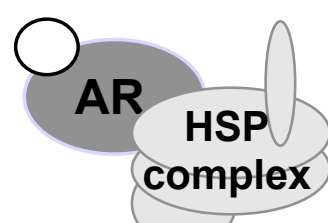

(1) Compete with ligand for AR binding
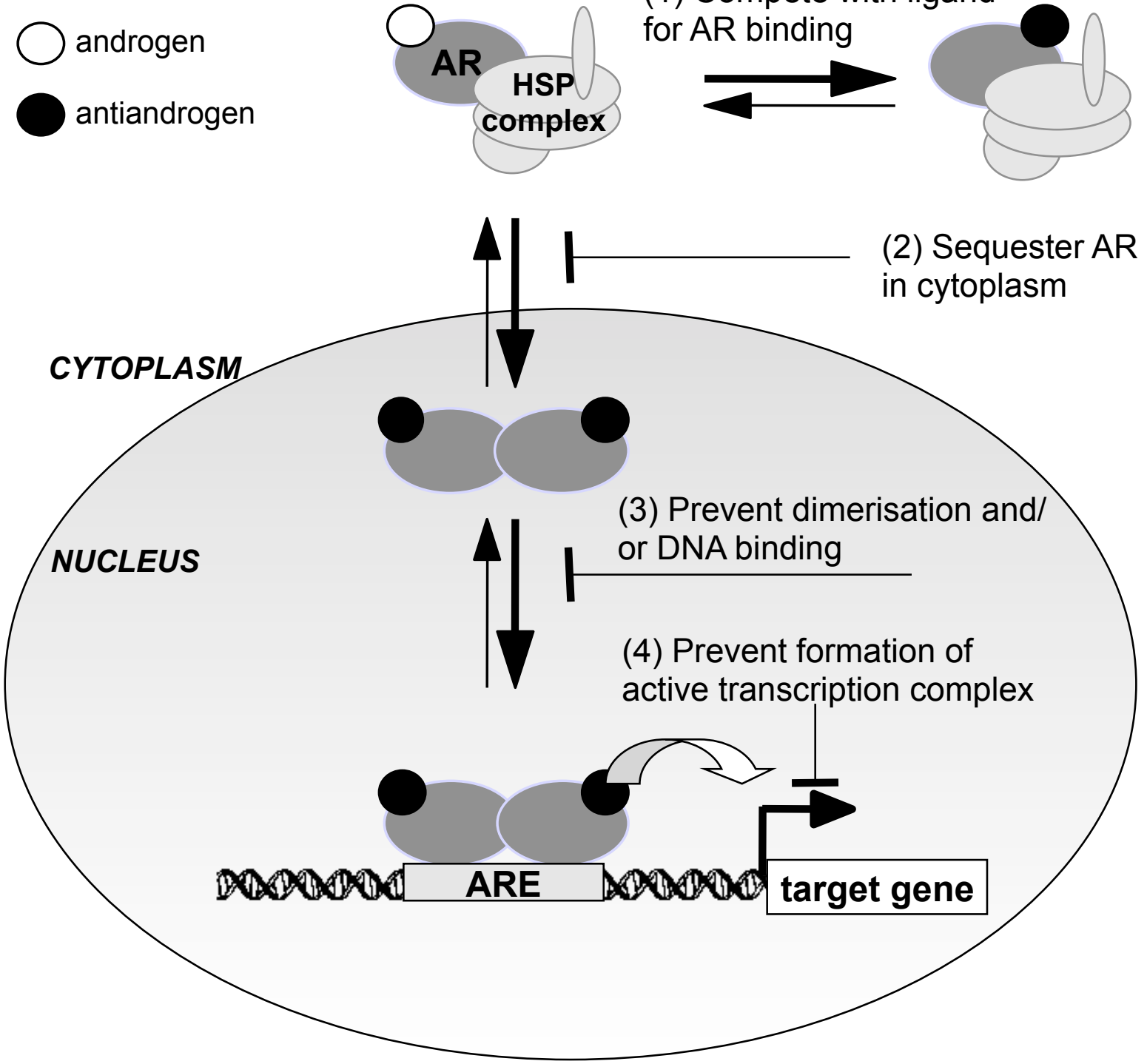
Figure 2

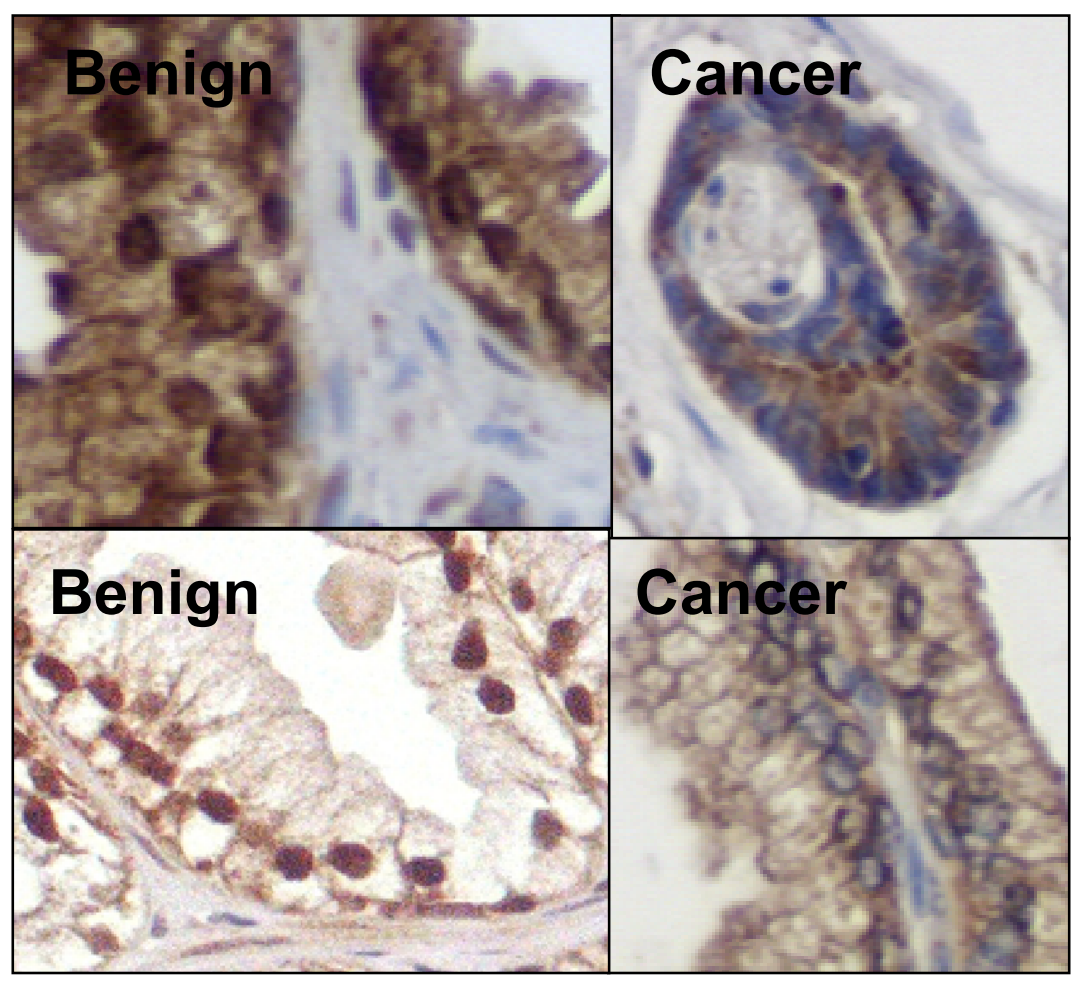

